\title{
Self-similar motion of laser fusion plasmas. Absorption in an unbounded plasma
}

\author{
A. Barrero and Juan R. Sanmartín \\ Escuela Tecnica Superior de Ingenieros Aeronautıcos, Universidad Poltécnica de Madrid, Madrid, Spain \\ (Received 28 July 1976, final manuscript received 21 January 1977) \\ The one-dimensional motion generated in a cold, infinte, uniform plasma of density $n_{0}$ by the absorption, \\ in a certain plane, of a linear pulse of energy per unit time and area $\phi=\phi_{0} t / \tau, 0<t \leq \tau$, is considered, the \\ analysis allows for thermal conduction and viscosity of 1ons and electrons, their energy exchange, and an \\ electron heat flux limiter The resulting motion is self-similar and governed by a single nondimensional \\ parameter $\alpha \propto\left(n_{0}^{2} \tau / \phi_{0}\right)^{2 / 3}$ Detalled asymptotic results are obtained for both $\alpha \ll 1$ and $\alpha \gg 1$, the general \\ behavior of the solution for arbitrary $\alpha$ is discussed The analysis can be extended to the case of a plasma \\ initially occupying a half-space, and throws light on how to optimize the hydrodynamics of laser fusion \\ plasmas Known approximate results corresponding to motion of a plasma submitted to constant irradiation \\ $(\phi)$ are recovered in the present work under appropriate limitıng processes
}

\section{INTRODUCTION}

To achieve break-even conditions in microfusion, a laser pulse must compress a DT pellet to densities well above that of the solid state, $n_{s}$, and strongly heat its core. ${ }^{1}$ This starts an outward burn wave that both minimizes the energy requirements and increases the burn efficiency. ${ }^{2}$

The cold, dense plasma around the core has a low specific entropy; consequently, the absorption of laser radiation must take place outside the dense pellet, since entropy production is associated with the absorption. This condition is met if the critical plasma density, $n_{\mathrm{er}}$ at the laser frequency, $\omega$, is smaller than $n_{s}$. Then, the radiation energy is deposited in an expanding corona of hot and rarefied plasma, that results from ablation of the pellet. ${ }^{3}$

Clearly, hydrodynamics must play an essential part in microfusion since it affects the attainment of the following goals: (a) the energy lost in the corona outflow should be held to a minimum; (b) entropy, and therefore entropy flux and production, should be kept low inside the pellet (except in its core); (c) both mass and energy should flow inward efficiently to reach high densities through compressional work.

The hydrodynamics of laser-plasma interaction has already been considered, both in the approximate, theoretical analysis, ${ }^{4-9}$ usually for constant irradiation, and as part of extensive computer simulations that include detailed fusion physics..$^{10-13}$ Nevertheless, exact results and clear conclusions for the hydrodynamic behavior of the plasma are still lacking.

In order to clarify how this behavior depends on the laser design parameters (maximum power flux $\phi_{0}$, pulse duration $\tau$, and frequency $\omega$ ) we consider the quasi-neutral motion of an initially cold and uniform plasma, allowing for viscosity and heat conduction of both electrons and ions, their energy exchange, and an electron heat flux limiter; other effects, like pressure radiation, nuclear fusion, emission (and re-absorption) of radiation, and non-Maxwellian distribution functions (a point discussed later) are not taken into account.
Then, a uniform power flux, linear in time (anomalously), absorbed in a certain plane, produces a self-similar motion. Anisimov ${ }^{14}$ noticed the existence of selfsimilar motion including electron conduction and ionelectron heating and Marshak ${ }^{15}$ pointed out self-similar motion of a gas with radiation heat conduction. We consider an unbounded plasma, as a step prior to the more difficult analysis of a half-space plasma, the case of interest for laser fusion plasmas, which we carry out in a forthcoming paper (any qualitative results found there should be valid for spherical geometries, except at the core of the pellet).

In Sec. II we discuss the equations and physics of the problem. Section III introduces both self-similar variables and nondimensional parameters. The resulting system of equations is analyzed in Secs. IV and V (Appendices $A$ and $B$ include some related mathematical details). Finally, the results obtained are summarized and discussed in Sec. VI.

\section{STATEMENT OF THE PROBLEM}

We consider a fully ionized, single ion-species plasma at rest, with uniform density $n_{0}$. At $t=0$, energy per unit time and unit area $\phi(t)$ starts being deposited at the plane $x=0$, and the plasma becomes one-dimensional. The macroscopic equations of continuity, momentum, and entropy for species $j$ ( $e$, electrons, $i$, ions) are

$\frac{D n_{j}}{D t}=-n_{j} \frac{\partial v_{j}}{\partial x}, \quad\left(\frac{D}{D t} \equiv \frac{\partial}{\partial t}+v_{j} \frac{\partial}{\partial x}\right)$,

$m_{j} n_{j} \frac{D v_{j}}{D t}=-\frac{\partial}{\partial x}\left(n_{j} k T_{j}\right)+\frac{4}{3} \frac{\partial}{\partial x}\left(\mu_{j} \frac{\partial v_{j}}{\partial x}\right)+q_{j} n_{j} E+R_{j}$,

$n_{j} T_{j} \frac{D}{D t}\left(k \ln \frac{T_{j}^{3 / 2}}{n_{j}}\right)=\frac{\partial}{\partial x}\left(\chi_{j} K_{j} \frac{\partial T_{j}}{\partial x}\right)+\frac{4}{3} \mu_{j}\left(\frac{\partial v_{j}}{\partial x}\right)^{2}+H_{j}$,

where $m, q, n, v$, and $T$ are particle mass and charge, density, macroscopic velocity, and temperature, respectively, and $\mu$ and $K$ are the classical coefficients of viscosity and thermal conduction ${ }^{16,17}$

$$
\mu_{j}=\bar{\mu}_{j} T_{j}^{5 / 2}, \quad K_{j}=\bar{K}_{j} T_{j}^{5 / 2} \text {. }
$$


Both $\bar{\mu}$ and $\bar{K}$ depend weakly on $T$ and $n$ through Coulomb logarithms.

We have assumed a collision-dominated plasma; that is, at each time $t$ the mean free path $\lambda$ and the time between collisions, for each species, are assumed to be much less than the length of plasma disturbed, $x_{f}(t)$ and $t$, respectively; the conditions implied by this hypothesis will be discussed in Sec. III. Nonetheless, a flux limiter $r^{12,18}$

$$
\chi_{e}=\left[1+\left(\frac{8 \pi m_{a}}{9 k^{2}}\right)^{1 / 2} \frac{\bar{K}_{e}}{n_{e}} T_{e}\left|\frac{\partial T_{e}}{\partial x}\right|\right]^{-1}
$$

is included in the electron heat conduction to approximately deal with situations where these conditions are not entirely met (viscous effects, for electrons, are much less important than thermal conduction even though viscous and thermal diffusivities are comparable, because, as will be shown, $v_{e} \simeq v_{i}$ and therefore, $m_{e} v_{e}^{2}$ $\left.\ll k T_{e}\right)$. On the other hand, since $\lambda_{j} \propto T_{j}^{2}$ and $\chi_{e}$ is only needed when $T_{t} \ll T_{\theta}$ (see Sec. IV), we set

$$
\chi_{i} \simeq 1
$$

ion diffusivities, moreover, are smaller than electron diffusivities by a factor of the order of $\left(m_{e} / m_{t}\right)^{1 / 2}$.

If the motion is quasi-neutral (a point to be discussed in Sec. III), $n_{e} \simeq n_{i} \equiv n$ for singly charged ions, and thus $(\partial / \partial x)\left(v_{e}-v_{i}\right) n \simeq 0$. Since $v_{j}$ vanishes in the undisturbed plasma, we have $v_{\varepsilon} \simeq v_{i} \equiv v$ everywhere. Then, Eqs. (1) become just

$$
\partial n / \partial t+\partial(n v) / \partial x=0 .
$$

Also, adding Eqs. (2) for $j=e, i$, neglecting $m_{e}$ against $m_{i}$, and making use of quasi-neutrality we find

$$
m_{i} n \frac{D v}{D t}=-\frac{\partial}{\partial x}\left[n k\left(T_{\theta}+T_{i}\right)\right]+\frac{4}{3} \frac{\partial}{\partial x}\left[\left(\mu_{\theta}+\mu_{i}\right) \frac{\partial v}{\partial x}\right]
$$

since obviously the ion-electron friction satisfies $R_{e}+R_{i}$ $=0$. Thus, the electric field $E$, set up by a slight charge separation, is dropped from the analysis.

Neither emission or absorption of radiation, nor nuclear fusion are considered here. The energy deposition at $x=0$ simulates the anomalous absorption of laser radiation, its pressure being neglected. Then,

$$
H_{i}=\frac{3}{2} n k\left[\left(T_{e}-T_{i}\right) / t_{e i}\right]
$$

and $H_{e}=-H_{i}+\phi(t) \delta(x), t_{e i}$ being the relaxation time for ion-electron energy exchange ${ }^{16}$

$$
t_{e l}=\bar{t}_{e l} T_{e}^{3 / 2} n^{-1},
$$

where $\bar{t}_{e l}$ involves a Coulomb logarithm.

If the laser absorption results in an energy per particle of the order of $0.1 \mathrm{keV}$ or larger, the present analysis would retain its validity even if we have a condensed substance, instead of a plasma, at $t=00^{4}$ For such energies we may, therefore, neglect the initial temperatures and have

$$
T_{e}(x, 0)=T_{1}(x, 0)=v(x, 0)=0, \quad n(x, 0)=n_{0} .
$$

On the other hand, because of the symmetry of the problem with respect to the plane $x=0$, we need only consid- er the $x>0$ half-space; then, the boundary conditions become

$$
\begin{aligned}
& T_{e}(\infty, t)=T_{i}(\infty, t)=v(\infty, t)=0, \quad n(\infty, t)=n_{0}, \\
& v(0, t)=0, \quad \partial T_{t} /\left.\partial x\right|_{x=0}=0 .
\end{aligned}
$$

Finally, integrating the electron entropy equation between $0^{-}$and $0^{*}$ we get

$$
2 \chi_{e} \bar{K}_{e} T_{e}^{5 / 2} \partial T_{e} /\left.\partial x\right|_{x=0}+\phi(t)=0 .
$$

Use of the boundary condition (12) allows one to drop the term $\phi(t) \delta(x)$ in $H_{e}$ and leads to

$$
H_{e}=-H_{i} \text {. }
$$

Now, if $\phi$ is linear

$$
\phi=\phi_{0} t / \tau, \quad 0<t \leqslant \tau,
$$

the solution of the system (6), (7), and (3), with Eqs. (8) and conditions (10)-(12), while the pulse is on, is self-similar (as shown in the next section) as long as the Coulomb logarithms in Eqs. (4), (5), and (9) can be approximated by constants.

\section{SELF-SIMILAR VARIABLES AND EQUATIONS}

Let

$$
\phi(t)=\phi_{0}(t / \tau)^{\phi} \quad 0<t \leqslant \tau
$$

and define

$$
\begin{aligned}
& \xi=x /\left[w t(t / \tau)^{a}\right], \quad n(x, t)=n_{0} \bar{n}(\xi), \\
& v(x, t)=v_{0}(t / \tau)^{r} u(\xi), \quad T_{j}(x, t)=T_{0}(t / \tau)^{s} \theta_{j}(\xi) .
\end{aligned}
$$

Introducing (13) and (14) into the equations of the last section, the powers of $t$ are found to drop out when

$$
p=1, \quad q=\frac{1}{3}, \quad r=\frac{1}{3}, \quad s=\frac{2}{3} .
$$

Now, since $w, v_{0}$, and $T_{0}$ may be chosen arbitrarily, we set

$$
\frac{v_{0}}{w}=\frac{4}{3}, \quad \frac{\bar{K}_{e} T_{0}^{5 / 2}}{k n_{0} w^{2} \tau}=1, \quad \frac{\bar{K}_{e} T_{0}^{7 / 2}}{\phi_{0} w \tau}=1,
$$

in order to simplify the continuity equation in the usual way, and take into account the fact that both energy deposition and electron heat conduction lie at the root of the phenomenon studied. Then, defining

$$
\alpha=3 k T_{0} / m_{i} v_{0} w,
$$

we can compute all nondimensional coefficients in the equations in terms of $\alpha$ and $A_{i}$ (the ion mass number) from the known values of $\bar{K}_{i}, \bar{\mu}_{j}$, and $\bar{t}_{e t}{ }^{16,17}$

We thus arrive at the following system:

$$
\begin{aligned}
& \frac{d \bar{n}}{d \xi}=\frac{\bar{n}}{\xi-u} \frac{d u}{d \xi}, \\
& u+4(u-\xi) \frac{d u}{d \xi}=-\frac{\alpha}{\bar{n}} \frac{d}{d \xi}\left[\vec{n}\left(\theta_{e}+\theta_{i}\right)\right] \\
& +\frac{1}{\bar{n}} \frac{d}{d \xi}\left[\left(\frac{0.039}{A_{i}^{1 / 2}} \theta_{i}^{5 / 2}+\frac{0.00049}{A_{i}} \theta_{e}^{5 / 2}\right) \frac{d u}{d \xi}\right],
\end{aligned}
$$




$$
\begin{aligned}
& \bar{n}\left[\theta_{e}\left(1+\frac{4}{3} \frac{d u}{d \xi}\right)+2(u-\xi) \frac{d \theta_{e}}{d \xi}\right]=\frac{d}{d \xi}\left(x_{e} \theta_{e}^{5 / 2} \frac{d \theta_{e}}{d \xi}\right) \\
& \quad+\frac{0.00065}{A_{i} \alpha} \theta_{e}^{5 / 2}\left(\frac{d u}{d \xi}\right)^{2}-4.3 \alpha \bar{n}^{2} \frac{\theta_{e}-\theta_{i}}{\theta_{e}^{3 / 2}}, \\
& \bar{n}\left[\theta_{i}\left(1+\frac{4}{3} \frac{d u}{d \xi}\right)+2(u-\xi) \frac{d \theta_{i}}{d \xi}\right]=\frac{0.041}{A_{i}^{1 / 2}} \frac{d}{d \xi}\left(\theta_{i}^{5 / 2} \frac{d \theta_{i}}{d \xi}\right) \\
& \quad+\frac{0.052}{A_{i}^{1 / 2} \alpha} \theta_{i}^{5 / 2}\left(\frac{d u}{d \xi}\right)^{2}+4.3 \alpha \bar{n}^{2} \frac{\theta_{e}-\theta_{i}}{\theta_{e}^{3 / 2}}, \\
& \chi_{e}=\left(1+\frac{0.059}{A_{i}^{1 / 2} \alpha^{1 / 2}} \frac{\theta_{e}}{\bar{n}}\left|\frac{d \theta_{e}}{d \xi}\right|\right)^{-1}
\end{aligned}
$$

with boundary conditions

$$
\begin{aligned}
& \theta_{e}(\infty)=\theta_{i}(\infty)=u(\infty)=0, \quad \bar{n}(\infty)=1, \\
& u(0)=0, \quad d \theta_{\imath} /\left.d \xi\right|_{\xi=0}=0, \quad 2 \chi_{e} \theta_{e}^{5 / 2} d \theta_{e} /\left.d \xi\right|_{\xi=0}=-1 .
\end{aligned}
$$

We have taken all the Coulomb logarithms equal to a constant of arbitrary value. The relative importance of electron heat conduction, ion viscosity and heat conduction, and electron viscosity is clear from the numerical coefficients in system (16).

It proves convenient to write Eqs. (16) as conservation laws:

$$
\begin{aligned}
& \bar{n}=\frac{d}{d \xi}[\bar{n}(\xi-u)], \\
& 5 \bar{n} u=\frac{d}{d \xi}\left[4 \bar{n} u(\xi-u)-\alpha \bar{n}\left(\theta_{e}+\theta_{i}\right)\right. \\
& \left.+\frac{0.039}{A_{l}^{1 / 2}} \theta_{i}^{5 / 2} \frac{d u}{d \xi}+\frac{0.00049}{A_{i}} \theta_{e}^{5 / 2} \frac{d u}{d \xi}\right], \\
& 3 \bar{n}\left(\theta_{e}+\theta_{i}+\frac{4 u^{2}}{3 \alpha}\right)=\frac{d}{d \xi}\left[2 \vec{n}(\xi-u)\left(\theta_{e}+\theta_{i}+\frac{4 u^{2}}{3 \alpha}\right)-\frac{4}{3} \vec{n} u\left(\theta_{e}+\theta_{i}\right)\right. \\
& +\chi_{e} \theta_{e}^{5 / 2} \frac{d \theta_{e}}{d \xi}+\frac{0.041}{A_{i}^{1 / 2}} \theta_{i}^{5 / 2} \frac{d \theta_{i}}{d \xi}+\frac{0.026}{A_{i}^{1 / 2}} \theta_{i}^{5 / 2} \frac{d u^{2}}{d \xi} \\
& \left.+\frac{0.00033}{A_{i}} \theta_{e}^{5 / 2} \frac{d u^{2}}{d \xi}\right],
\end{aligned}
$$

the last equations being a combination of Eqs. (16c) and $(16 \mathrm{~d})$. If $\xi_{f}$ is the length of plasma disturbed, we also get

$$
\begin{aligned}
& \int_{0}^{\xi_{f}} \bar{n} d \xi=\xi_{f}, \\
& \int_{0}^{\xi_{f}} 5 \bar{n} u d \xi=\left.\alpha \bar{n}\left(\theta_{e}+\theta_{i}\right)\right|_{\xi=0}, \\
& \int_{0}^{\xi_{f}} 3 \bar{n}\left(\theta_{e}+\theta_{i}+\frac{4 u^{2}}{3 \alpha}\right) d \xi=\frac{1}{2} .
\end{aligned}
$$

It is of interest to refer the parameters $n_{0}, \phi_{0}$, and $\tau$ to values typical of laser fusion plasmas. Choosing

$$
\begin{aligned}
& n_{r}=n_{s}(D T) \simeq 4.7 \times 10^{22} \mathrm{~cm}^{-3}, \quad \phi_{r}=10^{14} \mathrm{~W} \mathrm{~cm}^{-2}, \\
& \tau_{r}=10^{-9} \mathrm{sec}, \\
& \text { defining } \\
& \quad \hat{n}=n_{0} / n_{r}, \quad \hat{\phi}=\phi_{0} / \phi_{r}, \quad \hat{\tau}=\tau / \tau_{r},
\end{aligned}
$$

and setting the Coulomb logarithms equal to 8 , we then obtain

$$
\begin{aligned}
& \alpha=\frac{9 k}{4 m_{i}}\left(\frac{k^{2} n_{0}^{2} \tau}{\phi_{0} \bar{K}_{e}}\right)^{2 / 3} \simeq \frac{12.9}{A_{i}}\left(\frac{\hat{n}^{2} \hat{\tau}}{\hat{\phi}}\right)^{2 / 3}, \\
& v_{0}=\frac{4}{3} w=\frac{4}{3}\left(\frac{\phi_{0}^{5} \bar{K}_{e}^{2}}{k^{7} n_{0}^{7} \tau^{2}}\right)^{1 / 9} \simeq 1.73 \times 10^{7}\left(\frac{\hat{\phi}^{5}}{\hat{n}^{7} \hat{\tau}^{2}}\right)^{1 / 9} \mathrm{~cm} / \mathrm{sec}, \\
& T_{0}=\left(\frac{\phi_{0}^{2} \tau}{\bar{K}_{e} k n_{0}}\right)^{2 / 9} \simeq 1.05\left(\frac{\hat{\phi}^{2} \hat{\tau}}{\hat{n}}\right)^{2 / 9} \mathrm{keV} .
\end{aligned}
$$

The energy deposited in the plasma per unit area is

$$
\int_{0}^{\tau} \phi_{0}(t / \tau) d t \simeq 50 \hat{\phi} \hat{\tau} \mathrm{kJ} / \mathrm{cm}^{2} .
$$

For a sphere of radius $500 \mu$, that energy per area leads to a total energy

$$
E \simeq 1.57 \hat{\phi} \hat{\tau} \mathrm{kJ}
$$

The self-similar variables make a discussion of the approximations used in Sec. II easy. First, Eq. (17) shows that $\chi_{e} \simeq 1$ if

$$
\left(\frac{m_{e}}{\alpha m_{i}}\right)^{1 / 2} \frac{\theta_{e}}{\bar{n}}\left|\frac{d \theta_{e}}{d \xi}\right| \ll 1 .
$$

From the results in the following sections it may be shown that (24) is only violated for $\alpha$ comparable to $m_{e} /$ $m_{i}$ or less; thus, $x_{e} \simeq 1$, if $\alpha \gg m_{e} / m_{i}\left(\sim \frac{i}{5000}\right.$ for DT). A similar condition results from the requirement $\lambda \ll x_{f}$ $\equiv \xi_{f} w t(t / \tau)^{1 / 3} ;$ since $\lambda_{f} \sim \bar{K}_{f} T_{f}^{5 / 2}\left(m_{f} / k T_{f}\right)^{1 / 2}(k n)^{-1}$, we obtain, for electrons,

$$
\left(\alpha m_{i} / m_{e}\right)^{1 / 2} \gg \theta_{e}^{2} / \bar{n} \xi_{f} .
$$

Likewise, the time between electron collisions is much smaller than $t$ if

$$
\alpha m_{i} / m_{e} \gg \theta_{e}^{3 / 2} / \bar{n} \text {. }
$$

The corresponding inequalities for ions are found to be less restrictive.

Finally, the quasi-neutrality condition, $\lambda_{D} \equiv\left(k T_{e}\right)$ $\left.4 \pi n e^{2}\right)^{1 / 2} \ll x_{f}$, written in self-similar variables becomes

$$
\left(\theta_{e} \alpha\right)^{1 / 2} \xi_{f}^{-1} \ll\left(\omega_{p i} \tau\right) t / \tau,
$$

where $\omega_{p t}$ is the ion plasma frequency. It is thus clear that charge separation is not self-similar. Using expressions (21)-(23a), we obtain, from (25),

$$
\frac{\theta_{e}^{1 / 2} \alpha^{1 / 8}}{\bar{n}^{1 / 2} \xi_{f}} \frac{A_{t}^{1 / 8}}{\hat{\tau}^{3 / 4} \hat{\phi}^{1 / 4}} \ll 10^{5} \frac{t}{\tau} .
$$

The results for $\theta_{e}, \bar{n}$, and $\xi_{f}$ to be found later show that this inequality is easily satisfied for all reasonable values of $\alpha, A_{1}, \hat{\tau}$, and $\hat{\phi}$.

\section{ASYMPTOTIC SOLUTION FOR $\alpha \ll 1$}

Expanding all variables in powers of $\alpha$

$$
\begin{aligned}
& \bar{n}=\bar{n}_{1}+\alpha \bar{n}_{2}+\cdots, \\
& u=u_{1}+\alpha u_{2}+\cdots, \\
& \theta_{j}=\theta_{f 1}+\alpha \theta_{f 2}+\cdots,
\end{aligned}
$$

Eqs. (16) yield, to lowest order,

$$
\bar{n}_{1}=1, \quad u_{1}=\theta_{i 1}=0
$$




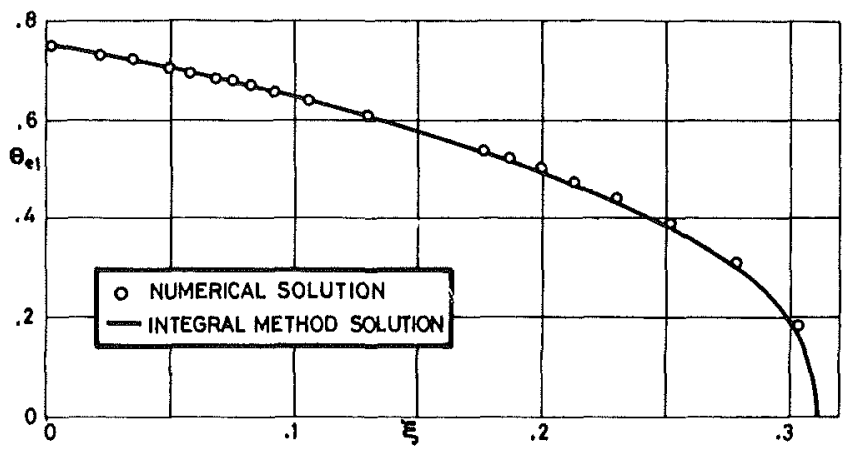

FIG. 1. Dimensionless electron temperature, $\theta_{01}$, vs dimensionless distance, $\xi$, for $\alpha \ll 1$.

and, assuming $1 \gg \alpha \gg m_{e} / m_{1}\left(\chi_{e} \simeq 1\right.$ except in a narrow neighborhood of $\xi_{f}$ ),

$$
\theta_{e 1}-2 \xi \frac{d \theta_{\sigma 1}}{d \xi}=\frac{d}{d \xi}\left(\theta_{e 1}^{5 / 2} \frac{d \theta_{e 1}}{d \xi}\right)
$$

with the boundary conditions

$$
\theta_{e 1}(\infty)=0, \quad \theta_{e 1}^{5 / 2} d \theta_{e 1} /\left.d \xi\right|_{t=0}=-\frac{1}{2} .
$$

Equation (26) represents a self-similar thermal wave. ${ }^{19}$ It may be shown that there exists a value, $\xi_{f}$, such that $\theta_{e 1} \equiv 0$ for $\xi \geqslant \xi_{f}$. Then, $\theta_{e 1}\left(\xi_{f}\right)=0, \xi_{f}$ being given by

$$
\int_{0}^{\xi} \theta_{e 1} d \xi=\frac{1}{8}
$$

this condition results from integrating (26) between 0 and $\xi_{f}$ and making the heat flux vanish at $\xi_{f}$. Figure 1 shows $\theta_{e 1}(\xi)$ as given both by a numerical computation and by an approximate, integral method solution ${ }^{20}$

$$
\theta_{e 1}=0.76(1-\xi / 0.31)^{0.41}, \quad \xi \leqslant 0.31 ;
$$

actually, $\theta_{e 1}$ behaves as $\left(\xi_{f}-\xi\right)^{0,4}$ near $\xi_{f}$.

The equations for $\bar{n}_{2}, u_{2}$, and $\theta_{i 2}$, neglecting viscosities and ion conduction, are

$$
\begin{aligned}
& d \bar{n}_{2} / d \xi=\xi^{-1} d u / d \xi, \\
& u_{2}-4 \xi d u_{2} / d \xi=-d \theta_{e 1} / d \xi, \\
& \theta_{12}-2 \xi d \theta_{12} / d \xi=4.3 \theta_{e 1}^{-1 / 2},
\end{aligned}
$$

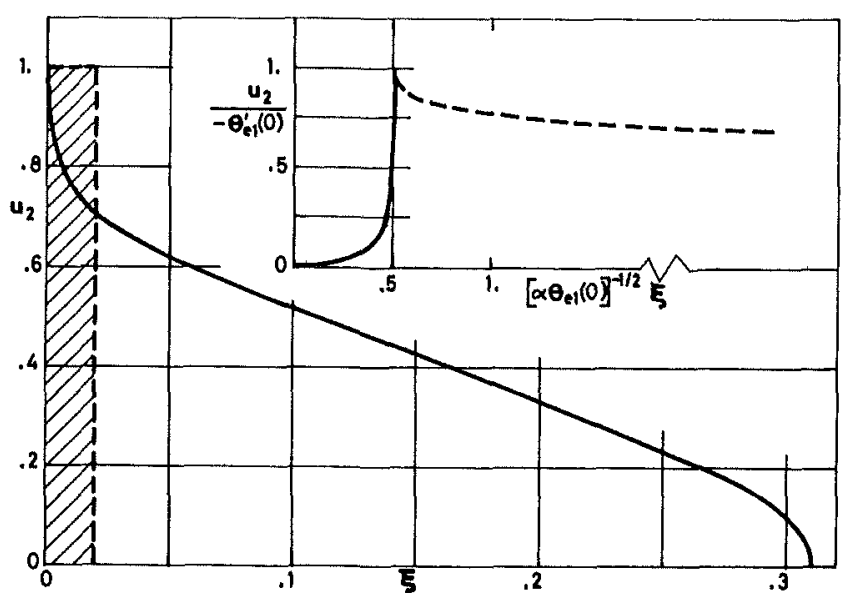

FIG. 2. Dimensionless velocity, $u_{2}$, vs dimensionless distance, $\xi$, for $\alpha \ll 1$. [The insert represents $u_{2}$ vs $\xi$ in the inner layer, $\left.\xi=0\left(\alpha^{1 / 2}\right)\right]$.

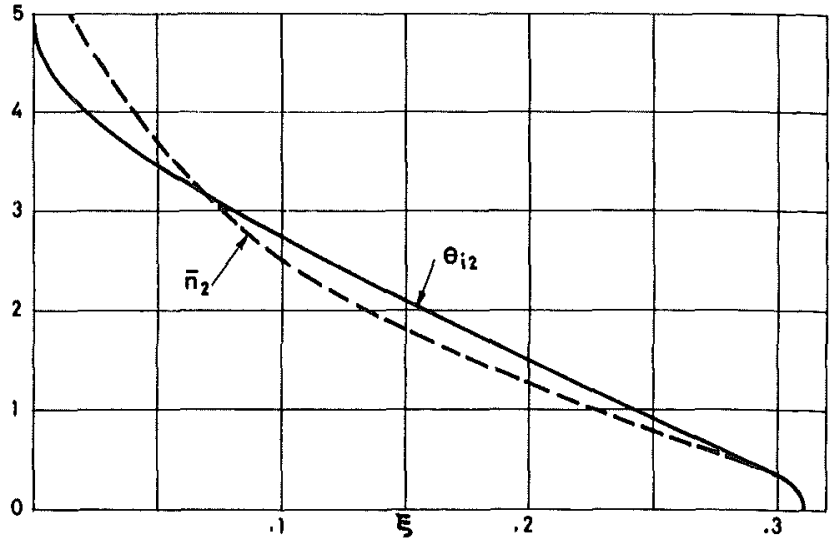

FIG. 3, Dimensionless density perturbation, $\bar{n}_{2}$, and ion temperature, $\theta_{i 2}$, vs dimensionless distance, $\xi$, for $\alpha \ll 1$.

with boundary conditions

$$
\begin{aligned}
& \bar{n}_{2}\left(\xi_{f}\right)=u_{2}\left(\xi_{f}\right)=\theta_{i 2}\left(\xi_{f}\right)=0, \\
& u_{2}(0)=0, \quad d \theta_{i 2} /\left.d \xi\right|_{\zeta=0}=0 .
\end{aligned}
$$

We then have

$$
\begin{aligned}
& u_{2}=-\xi^{1 / 4} \int_{\xi}^{\xi_{f}}\left(d \theta_{e 1} / d \xi_{1}\right) d \xi_{1} / 4 \xi_{1}^{-5 / 4}, \\
& \bar{n}_{2}=\int_{\xi}^{\xi_{f}}\left(d u_{2} / d \xi_{1}\right) d \xi_{1} / \xi_{1}, \\
& \theta_{f 2}=2.15 \xi^{1 / 2} \int_{\xi}^{\xi_{f}} d \xi_{1} / \theta_{e 1}^{1 / 2} \xi_{1}^{3 / 2} ;
\end{aligned}
$$

these solutions are represented in Figs. 2 and 3.

For small $\xi$

$$
\begin{aligned}
& u_{2} \simeq-\theta_{e 1}^{\prime}(0)-C \xi^{1 / 4}, \\
& \theta_{i 2} \simeq 4.3\left[\theta_{e 1}(0)\right]^{-1 / 2}-C_{1} \xi^{-1 / 2}, \\
& \bar{n}_{2} \simeq C_{2} \xi^{-3 / 4},
\end{aligned}
$$

where $\theta_{e 1}^{\prime} \equiv d \theta_{e 1} / d \xi$ and $C, C_{1}, C_{2}$ are constants; thus, expressions $(29 \mathrm{a}, \mathrm{c})$ do not satisfy conditions $(28 \mathrm{~b})$, while $\bar{n}_{2}$ becomes singular at $\xi=0$. Clearly, a thin, inner layer must exist around $\xi=0$, in which some terms neglected in (27) should be retained.

\section{A. Inner layer}

Defining new, stretched variables

$$
\hat{\xi}=\xi / \alpha^{1 / 2}\left[\theta_{e_{1}}(0)\right]^{1 / 2}, \quad \hat{u}=-u_{2} / \theta_{\theta_{1}}^{\prime}(0),
$$

Eq. (16b) becomes

$$
\hat{u}-1=\left(4 \hat{\xi}-\hat{\xi}^{-1}\right) d \hat{u} / d \hat{\xi}
$$

whose solution is

$$
\hat{u}=1-A\left|1-4 \hat{\xi}^{2}\right|^{1 / 8} \text {. }
$$

Boundary condition $\hat{u}(0)=0$ leads to $A=1$; on the other hand, matching (31) for large $\hat{\xi}$ to $(30 a)$, we get

$$
A=-\left[\alpha \theta_{e 1}(0) / 4\right]^{1 / 8} C / \theta_{e 1}^{\prime}(0) \text {. }
$$

Thus, $\hat{u}$ presents a cusp at $\hat{\xi}=1 / 2$ (see Fig. 2).

The $\hat{\xi}=\frac{1}{2}$ plane is, therefore, a weak discontinuity 
surface. ${ }^{21}$ Such surfaces move relative to the fluid with the speed of propagation of small disturbances, $c$, which, due to heat conduction, is given here by $c^{2}$ $\equiv m_{i}^{-1}(d P / d n)_{T} \simeq k T_{e} / m_{i}\left(T_{i} \ll T_{e}\right) ;$ now, at $\hat{\xi}=\frac{1}{2}$ we have

$$
4(\xi-u)^{2} \simeq 4 \xi^{2}=\alpha \theta_{\beta}(0),
$$

or

$$
\left(d x_{d} / d t-v\right)^{2}=k T_{e} / m_{i},
$$

$x_{d}$ being the position of the discontinuity surface.

Obviously, there must exist a very thin, viscous sublayer, centered at $\hat{\xi}=\frac{1}{2}$; a detailed analysis of this sublayer is given in Appendix A.

We notice that $\bar{n}_{2}$, as given by $(29 \mathrm{~b})$ and $(31)$, is no longer singular at $\xi=0$. On the other hand, to satisfy condition $d \theta_{t 2} / d \xi=0$ at $\xi=0$, heat conduction should be retained in the ion energy equation, within a very thin, thermal, boundary layer around $\xi=0$.

\section{ASYMPTOTIC SOLUTION FOR $\alpha \gg 1$}

Condition $\alpha \ll 1$ implies either $n_{0}$, and thus, heat capacity per unit volume, small, for given $\phi_{0} / \tau$, or $\phi_{0} / \tau$ large for given $n_{0}$; either way $T_{e}$ must grow very fast with time. Heat conduction is then dominant, leading to a rapid equalization of both temperature and pressure; consequently, the plasma is unable to begin moving, and convection is negligible, as found in last section. In the opposite limit $\alpha \gg 1$, on the contrary, convective energy flow must be dominant and give rise (neglecting viscosity) to a shock bounding the disturbed plasma. ${ }^{19}$ However, since convection must vanish at $\xi=0$, there must exist a region where heat conduction is important, lying between the origin and the (isentropic) region where convection is dominant.

\section{A. Isentropic region}

The flow behind the shock is isentropic, ion and electron temperatures being equal and viscosity and conduction, negligible. The jump conditions across the shock, which may be directly obtained from system (19), are

$$
\begin{aligned}
& \bar{n}_{f}\left(\xi_{f}-u_{f}\right)=\xi_{f}, \\
& 4 \bar{n}_{f} u_{f}\left(\xi_{f}-u_{f}\right)-2 \alpha \bar{n}_{f} \theta_{f}=0, \\
& 4 \bar{n}_{f}\left(\theta_{f}+2 u_{f}^{2} / 3 \alpha\right)\left(\xi_{f}-u_{f}\right)-8 \bar{n}_{f} u_{f} \theta_{f} / 3=0,
\end{aligned}
$$

where the subscript $f$ labels the conditions just behind the shock; then,

$$
\bar{n}_{f}=4, \quad u_{f}=3 \xi_{f} / 4, \quad \theta_{f}=3 \xi_{f}^{2} / 8 \alpha .
$$

Defining the normalized variables

$$
\eta=\xi / \xi_{f}, \quad \nu=\bar{n} / \bar{n}_{f}, \quad y=u / u_{f}, \quad z_{j}=\theta_{j} / \theta_{f},
$$

Eq. (20c) becomes

$$
\int_{0}^{1} 3 \nu\left(z_{e}+z_{\uparrow}+2 y^{2}\right) d \eta=\alpha / 3 \xi_{f}^{3}
$$

since $\eta, \nu, y$, and $z_{f}$ are of order unity behind the shock, $\xi_{f}=\gamma \alpha^{1 / 3}$, where $\gamma$ is an unknown constant of order of unity, determining the shock position.

Then, system (16) becomes

$$
\begin{aligned}
& \frac{d \nu}{d \eta}=\frac{3 \nu}{4 \eta-3 y} \frac{d y}{d \eta}, \\
& \nu y+\nu(3 y-4 \eta) \frac{d y}{d \eta}=-\frac{1}{2} \frac{d}{d \eta}\left[\nu\left(z_{e}+z_{t}\right)\right]+O\left(\alpha^{-3 / 2}\right), \\
& \begin{aligned}
\nu\left[z_{j}\left(1+\frac{d y}{d \eta}\right)+\frac{1}{2}(3 y-4 \eta) \frac{d z_{j}}{d \eta}\right] \\
=\mp \frac{75 \alpha^{3 / 2} \nu^{2}}{\gamma^{3}} \frac{z_{e}-z_{i}}{z_{e}^{3 / 2}}+O\left(\alpha^{-3 / 2}\right),
\end{aligned}
\end{aligned}
$$

the upper sign corresponding to electrons. The viscosity and conduction terms are $O\left(\alpha^{-3 / 2}\right)$, as indicated, and may be neglected to lowest order. Similarly, we get $\chi_{\theta} \simeq 1$ up to order $\alpha^{-3 / 2}$. On the other hand, the energy exchange term is dominant, so that $z_{e} \simeq z_{i} \equiv z$. Thus, Eq. (32b) will read

$$
\nu y+\nu(3 y-4 \eta) d y / d \eta=-d(\nu z) / d \eta,
$$

while adding the (32c) equations for ions and electrons, we obtain

$$
2 z(1+d y / d \eta)+(3 y-4 \eta) d z / d \eta=0 \text {. }
$$

Equations (32a), (32d), and (32e) must be solved subject to boundary conditions

$$
\nu(1)=y(1)=z(1) .
$$

Having neglected second-order derivatives, that system will clearly not be uniformly valid in the whole interval $0<\eta \leqslant 1$.

A first integral of the system can be obtained by combining Eqs. (32a, e),

$$
\nu^{7 / 3}(4 \eta-3 y) / z^{2}=1
$$

this is the usual adiabatic integral of self-similar isentropic flow. ${ }^{22}$ Furthermore, Eq. (32d) may be rewritten as

$$
\frac{d y}{d \eta}=-\frac{y(4 \eta-3 y)+2 z}{5 z-(4 \eta-3 y)^{2}}
$$

This equation, together with Eq. (32e), may now be easily solved defining

$$
Y=y / \eta, \quad Z=z / \eta^{2}
$$

this leads to a $(Y, Z)$ phase-space equation

$\frac{d Z}{d Y}=\frac{2 Z}{4-3 Y} \frac{Z(17-15 Y)+(4-3 Y)[Y+3(Y-1)(4-3 Y)]}{Z(2+5 Y)+3 Y(Y-1)(4-3 Y)}$

together with

$$
\eta \frac{d Y}{d \eta}=-Y-\frac{Y(4-3 Y)+2 Z}{5 Z-(4-3 Y)^{2}} .
$$

It may be shown analytically, that $Y$ increases, starting from unity, as $\eta$ decreases and that for $Y \rightarrow 4 / 3, Z \sim(4$ $-3 Y)^{3 / 13}$; then, defining $\bar{\eta}$ by $Y(\bar{\eta})=4 / 3$, we obtain $z \simeq B_{1}(\eta-\bar{\eta})^{3 / 13}, \quad y \simeq \frac{4}{3} \bar{\eta}-\frac{2}{5}(\eta-\bar{\eta}), \quad \nu \simeq B_{2}(\eta-\bar{\eta})^{-3 / 13}$.

Numerical results for $\nu, y$, and $z$ versus $\eta$ are given in Fig. 4 ; we also find $\bar{\eta} \simeq 0.82, B_{1} \simeq 1.70, B_{2} \simeq 0.78$.

This solution corresponds to the classical problem of a gas at rest, compressed by a plane piston moving with a velocity $U \propto t^{n}$. For certain values of $n$, the density 


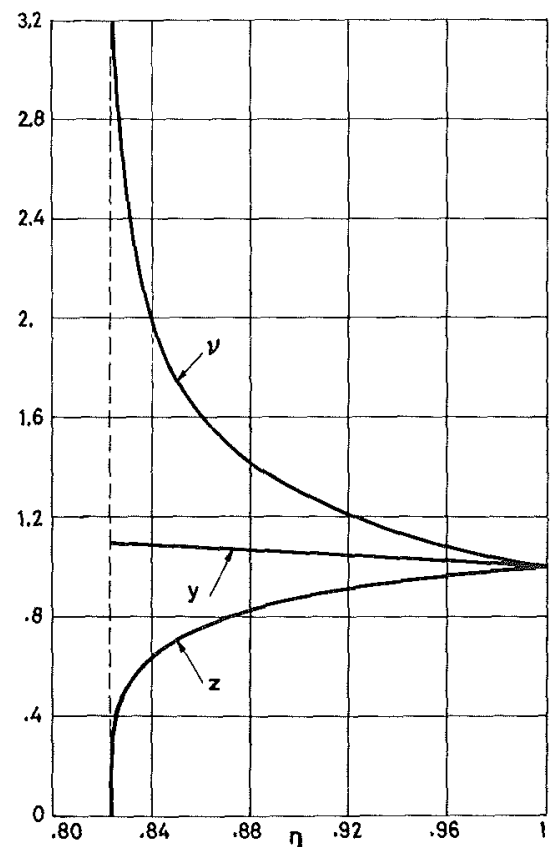

FIG. 4. Normalized dimensionless density, $\nu$, velocity, $y$, and ion and electron temperatures, $z$, vs normalized dimensionless distance, $\eta$; isentropic region, $\alpha \gg 1$.

becomes infinite at the piston, while the temperature goes to zero, as in the present case $(n=1 / 3) .^{22-24}$

\section{B. Conduction region}

The isentropic solution ceases to be valid in the neighborhood of $\bar{\eta}$. Moreover, in a region containing the origin, conduction must be taken into account. To determine the order of magnitude of the variables in this region we first notice that the last boundary condition in (18), may be rewritten

$$
(3 / 8)^{7 / 2} \gamma^{6} \alpha^{-3 / 2} \chi_{e} z_{e}^{5 / 2} d z_{e} /\left.d \eta\right|_{\eta=0}=-\frac{1}{2} ;
$$

thus, $z_{e}=O\left(\alpha^{3 / 7}\right)$, and it is easy to show that $z_{e} \simeq z_{i} \equiv z$, $\chi_{e} \simeq 1$. On the other hand, $y=O(1)$, since $y\left(\eta^{+}\right)$is of the order of unity. Finally, Eq. (20a) gives $\int_{0}^{1} \nu d \eta=1 / 4$, while the isentropic solution is such that $\int \frac{1}{\eta} \nu d \eta=1 / 4$; thus, $\nu$ must be much less than unity to the left of $\bar{\eta}$. Then, from $(20 \mathrm{~b})$ we get $\int \frac{1}{\eta} 5 \nu y d \eta \simeq v(0)_{z}(0)$, implying $\nu=O\left(\alpha^{-3 / 7}\right)$.

Now defining $\hat{\nu}=\alpha^{3 / 7} \nu, \hat{z}=\alpha^{-3 / 7} z$, system (16) to lowest order in $\alpha$ becomes

$\frac{d \hat{\nu}}{d \eta}=\frac{3 \hat{\nu}}{4 \eta-3 y} \frac{d y}{d \eta}$,

$d(\hat{\nu} \hat{z}) / d \eta=0$

$2 \hat{\nu} \hat{z}\left(1+\frac{d y}{d \eta}\right)-\hat{\nu}(4 \eta-3 y) \frac{d \hat{z}}{d \eta}=\left(\frac{3}{8}\right)^{5 / 2} \frac{\gamma^{3}}{4} \frac{d}{d \eta}\left(\hat{z}^{5 / 2} \frac{d \hat{z}}{d \eta}\right) ;$

Eq. (33c) results from adding $(16 c, d)$. We have neglected viscosities and ion conduction. System (33) must be solved subject to five boundary conditions, since $\gamma$ is unknown: three conditions for matching with the isentropic solution, and

$$
y(0)=0,\left.\quad\left(\frac{3}{8}\right)^{7 / 2} \gamma^{6} \hat{z}^{5 / 2} \frac{d \hat{z}}{d \eta}\right|_{\eta=0}=-\frac{1}{2} .
$$

Integrating (33b) and matching to the isentropic solution, we obtain

$$
\hat{\nu} \hat{z} \simeq B_{1} B_{2} \simeq 1.32 \text {. }
$$

Then, using (33a) and (34), Eq. (33c) may be integrated once, giving

$$
1.32(2 \eta+5 y)=(3 / 8)^{5 / 2}\left(\gamma^{3} / 4\right) \hat{z}^{5 / 2} d \hat{z} / d \eta+1 / 3 \gamma^{3} .
$$

Also, Eq. (33a) becomes

$$
3 \hat{z} d y / d \eta=-(4 \eta-3 y) d \hat{z} / d \eta \text {. }
$$

The last two equations are to be solved with boundary conditions

$$
y(0)=0, \quad y(\bar{\eta})=\frac{4}{3} \bar{\eta} \quad, \quad \hat{z}(\bar{\eta})=0 .
$$

In the neighborhood of $\vec{\eta}$, the solution is found to behave in the form

$$
\hat{z} \simeq D_{1}(\bar{\eta}-\eta)^{2 / 5}, \quad y \simeq \frac{4}{3} \bar{\eta}-D_{2}(\bar{\eta}-\eta)^{2 / 5} .
$$

Then, $\hat{z}^{5 / 2} d \hat{z} / d \eta \rightarrow 0$ as $\eta-\bar{\eta}$, and from Eq. (35)

$$
\gamma=0.33 \text {. }
$$

Numerical results for $\hat{\nu}, y$, and $\hat{z}$ versus $\eta$ are given in Fig. 5.

It is important to notice that expression $\nu(4 \eta-3 y)$ must increase monotonically with $\eta$, for all $\eta$ [see Eq. (19a)], and that the solution in this section violates this condition at $\bar{\eta}$ :

$\nu(4 \eta-3 y) \sim(\eta-\bar{\eta})^{10 / 13} \rightarrow 0$ as $\eta \rightarrow \bar{\eta}^{*}$,

$\nu(4 \eta-3 y) \sim \alpha^{-3 / 7}(\bar{\eta}-\eta)^{-2 / 5}(\bar{\eta}-\eta)^{2 / 5} \sim \alpha^{-3 / 7}$ as $\eta \rightarrow \bar{\eta}^{-}$,

and therefore, $\nu(4 \eta-3 y)$ (mass flow relative to the surface $\eta$ ) presents a negative jump at $\bar{\eta}$, of order $\alpha^{-3 / 7}$. A detailed analysis of the solution in the neighborhood of $\bar{\eta}$, carried out in Appendix B, shows that $\nu(4 \eta-3 y)$ is continuous at this point, and that $z$ and $\nu$ present a minimum $\left(\simeq 0.77 \alpha^{-9 / 70}\right)$ and a maximum $\left(\simeq 1.72 \alpha^{9 / 70}\right)$, respectively, at $\bar{\eta}+0.032 \alpha^{-39 / 70}$. Naturally, the solution given in Figs. 4 and 5 , will not be modified by this correction, except in a narrow layer around $\eta=\bar{\eta}$.

\section{DISCUSSION OF RESULTS}

We have studied the one-dimensional motion generated in a cold, unbounded plasma of density $n_{0}$, when a pulse of energy per unit time and area, $\phi=\phi_{0} t / \tau$, is (anomalously) absorbed in a given plane. The analysis, includ-

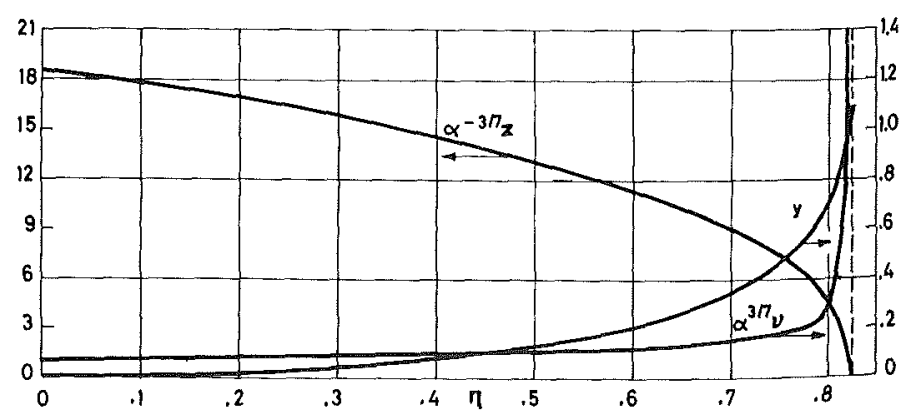

FIG. 5. Normalized dimensionless density, $\nu$, velocity, $y$, and ion and electron temperatures, $z$, vs normalized dimensionless distance, $\eta$; conduction region, $\alpha \gg 1$. 


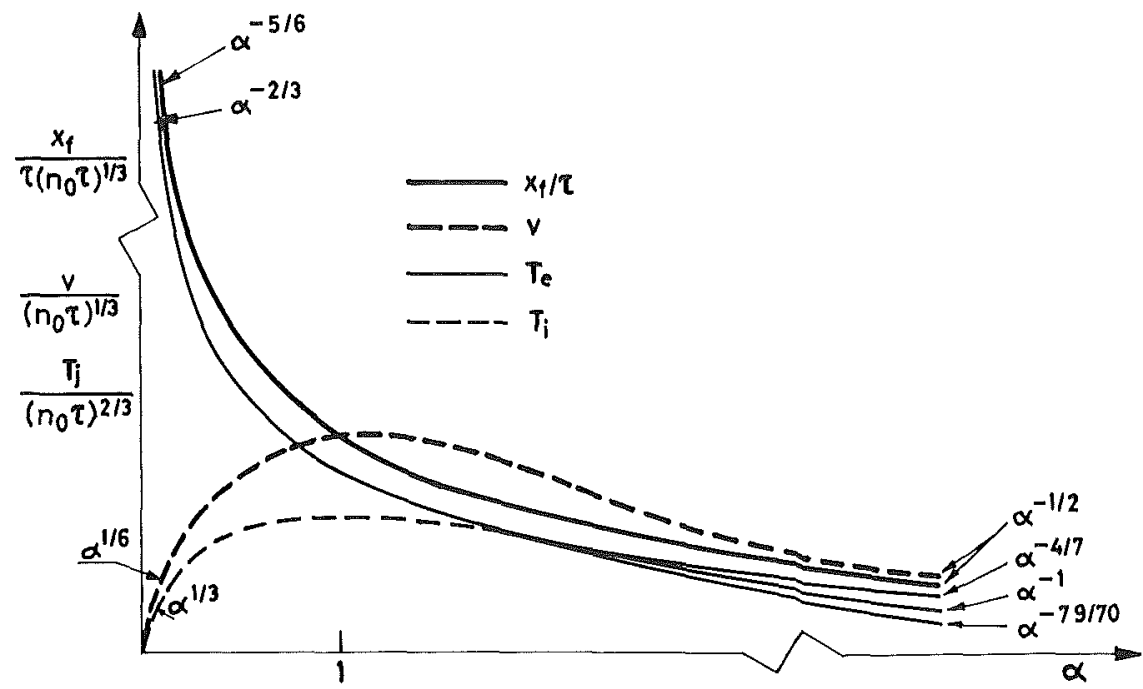

FIG. 6. Schematic dependence of length of plasma disturbed, velocity and temperatures as functions of $n_{0}, \tau$, and $\alpha$. ing heat conduction, viscosity, ion-electron energy exchange, and an electron flux limiter, shows that the motion is self-similar and governed by a single parameter $\alpha \propto\left(n_{0}^{2} \tau / \phi_{0}\right)^{2 / 3}$. For all reasonable values of $n_{0}, \tau$, and $\phi_{0}$, the motion is quasi-neutral, and for $\alpha \gg m_{e} / m_{i}$ the plasma is collision dominated (no flux limiter).

For $\alpha \ll 1$, a thermal wave carries the energy, convection and ion temperature being negligible. The order of magnitude of the self-similar variables defined in $\mathrm{Sec}$. III is

$$
\xi_{f} \sim \theta_{e}=O(1), \quad \theta_{i} \sim \bar{n}-1 \sim u=O(\alpha) .
$$

Both $\bar{n}$ and $u$ present a maximum for $\xi=O\left(\alpha^{1 / 2}\right)$.

For $\alpha \gg 1$, ion and electron temperatures are practically equal to each other. Close to the origin, there is a region where conduction and thermal energy convection are comparable; a thin layer of cold and very dense plasma separates it from an isentropic region further ahead, where thermal and kinetic energies are of the same order, and which is bounded by a shock from the undisturbed plasma. The order of magnitude of the self-similar variables is

Conduction region:

$$
\bar{n}=O\left(\alpha^{-3 / 7}\right), \quad \theta_{j}=O\left(\alpha^{2 / 21}\right), \quad \Delta \xi=O\left(\alpha^{1 / 3}\right) ;
$$

Isentropic region:

$$
\bar{n}=O(1), \quad \theta_{j}=O\left(\alpha^{-1 / 3}\right), \quad \Delta \xi=O\left(\alpha^{1 / 3}\right) ;
$$

Intermediate layer:

$$
\bar{n}=O\left(\alpha^{8 / 70}\right), \quad \theta_{j}=O\left(\alpha^{-97 / 210}\right), \quad \Delta \xi=O\left(\alpha^{-47 / 210}\right) ;
$$

where $\Delta \xi$ is the thickness of the region considered; $u=O\left(\alpha^{1 / 3}\right)$ everywhere. There is also a shock precursor ${ }^{19}$ of thickness $\Delta \xi=O\left(\alpha^{-5 / 6}\right)$.

Qualitative information on the solution for $\alpha=O(1)$ may be obtained from the results for $\alpha \ll 1$ and $\alpha \gg 1$. For $\alpha$ large and decreasing, the density maximum and temperature minimum become less sharp, while $\Delta \xi$, for all zones, approaches $O(1)$. Thus for $\alpha=O(1)$, a shock will stand in the middle of a thermal wave; in other words, the precursor thickness and the distance of the shock to the origin are comparable and of order of unity. Then, convection and conduction of energy are comparable everywhere; moreover, $\theta_{e}, \theta_{i}, \bar{n}$, and $u$ are of order unity, and $\theta_{e} \neq \theta_{i}$. As $\alpha$ decreases further the shock collapses toward the origin until, at a certain $\alpha$, becomes a weak discontinuity surface, where $\bar{n}$ and $u$ present a maximum $(\alpha \ll 1$ case $)$.

The dimensional variables $x_{f}, v$, and $T,(j=e, i)$, at $t=\tau$, depend on $\alpha$ in the way

$$
\begin{aligned}
& x_{f} / \tau \propto\left(n_{0} \tau\right)^{1 / 3} \xi_{f}(\alpha) \alpha^{-5 / 6} \propto\left(\phi_{0} \tau\right)^{1 / 6} \xi_{f}(\alpha) \alpha^{-7 / 12}, \\
& v \propto\left(n_{0} \tau\right)^{1 / 3} u(\alpha, \xi) \alpha^{-5 / 6} \propto\left(\phi_{0} \tau\right)^{1 / 6} u(\alpha, \xi) \alpha^{-7 / 12}, \\
& T_{j} \propto\left(n_{0} \tau\right)^{2 / 3} \theta_{j}(\alpha, \xi) \alpha^{-2 / 3} \propto\left(\phi_{0} \tau\right)^{1 / 3} \theta_{j}(\alpha, \xi) \alpha^{-1 / 6} .
\end{aligned}
$$

These expressions are schematically represented in Figs. 6 and 7; for large $\alpha, T$, presents different behavior depending on the value of $\xi$.

We next point out some qualitative conclusions of interest for laser fusion plasmas:

(a) Both velocity (and therefore kinetic energy) and ion temperature, as function of $\alpha$, present a maximum for a value of $\alpha$ of order unity.

(b) Both production and flux of entropy depend critically on $\alpha$ since, first, $T_{e} \simeq T_{i}$ for $\alpha \gg 1$ and $T_{e} \neq T_{i}$ for $\alpha \leqslant O(1)$, and second, for $\alpha \gg 1$ the motion of the plas$m a$ in the front is is entropic, while for $\alpha \leqslant O(1)$ heat conduction is important everywhere.

We notice here that these conclusions will remain valid for spherical geometries and for nonlinear pulses (if appropriate values of $d \phi / d t$ are used instead of $\left.\phi_{0} / \tau\right)$.

Finally, some approximate results by previous authors may be recovered as certain limits of our solution:

(1) For given $\phi_{0}$ and $t \simeq \tau \rightarrow \infty\left(\phi \simeq \phi_{0}, \phi / t-0\right), \alpha \rightarrow \infty$, and therefore

$$
x_{f} / \tau \sim v \sim\left(\phi_{0} / n_{0}\right)^{1 / 3}, \quad T_{e} \simeq T_{i} \sim\left(\phi_{0} / n_{0}\right)^{2 / 3} .
$$

This behavior has been previously obtained in Refs. 6 and 7, for constant irradiation $\phi=\phi_{0}$, and long times (neglecting any transient) so that $\phi / t \rightarrow 0$. 


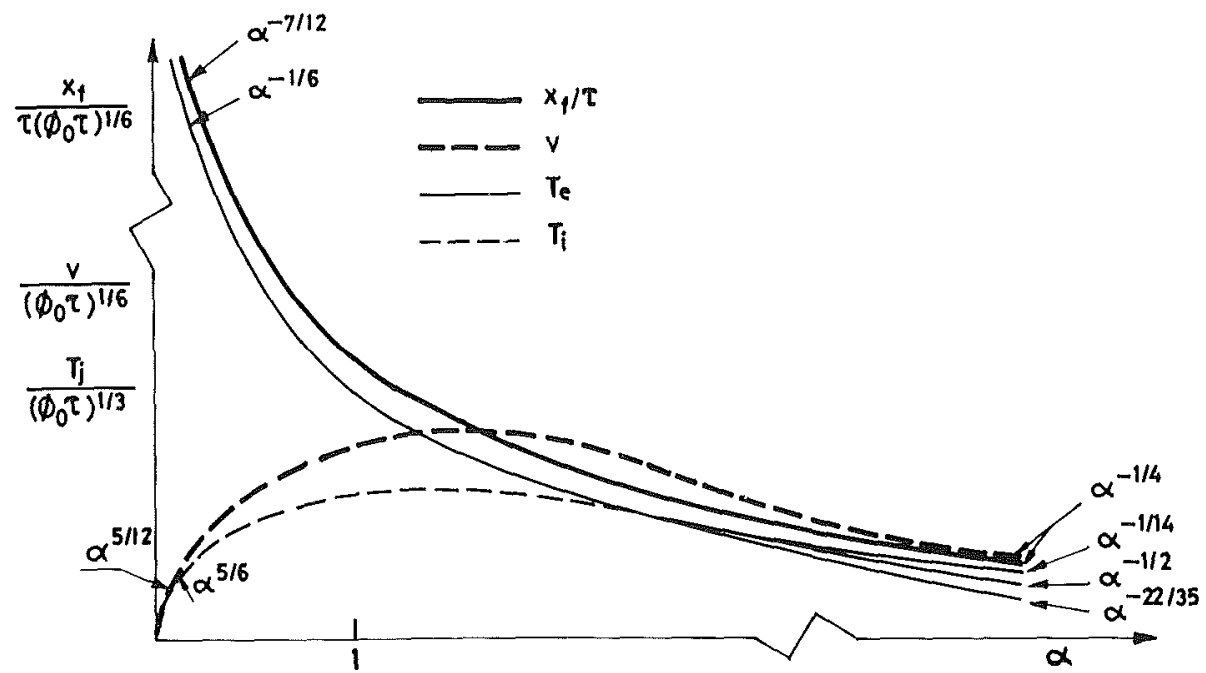

FIG. 7. Schematic dependence of length of plasma disturbed, velocity and temperatures as functions of $\phi_{0}, \tau$, and $\alpha$.

(2) For given $\phi_{0}$ and $t \simeq \tau \rightarrow 0\left(\phi \simeq \phi_{0}, \phi / t \rightarrow \infty\right), \alpha \rightarrow 0$, and therefore,

$$
x_{f} \sim\left(\phi_{0}^{5} \tau^{7} / n_{0}^{7}\right)^{1 / 9}, \quad T_{e} \sim\left(\phi_{0}^{2} \tau / n_{0}\right)^{2 / 9} .
$$

This behavior has been previously obtained in Refs. 8 and 9 , for constant irradiation $\phi=\phi_{0}$, and short times (neglecting motion) so that $\phi_{0} / t \rightarrow \infty$. One may also consider the instantaneous deposition of energy per unit area $\dot{W} \equiv \phi_{0} \tau$; this leads to

$$
x_{f} \sim\left(\dot{W}^{5} \tau^{2} / n_{0}^{7}\right)^{1 / 9}, \quad T_{e} \sim\left(\dot{W}^{2} / \tau n_{0}\right)^{2 / 9} .
$$

\section{ACKNOWLEDGMENTS}

This work was conducted at the Universidad Politécnica de Madrid, Spain, in partial fulfillment of the requirements for the doctoral degree of one of the authors (A.B.).

This research was performed under the auspices of the Junta de Energía Nuclear of Spain.

\section{APPENDIX A}

To study the viscous sublayer around $\hat{\xi}=1 / 2$, for $\alpha \ll 1$, we define

$$
\xi^{*}=(\hat{\xi}-1 / 2)\left[\alpha \theta_{e 1}(0) / \sigma\right]^{1 / 2}, \quad u^{*}=1-\hat{u},
$$

where

$$
\sigma=0.039\left[\theta_{i 1}(0) \alpha\right]^{5 / 2} / A_{i}^{1 / 2}+0.00049\left[\theta_{e 1}(0)\right]^{5 / 2} / A_{i} .
$$

Then, the momentum equation becomes

$$
\frac{d^{2} u^{*}}{d \xi^{* 2}}+8\left[\xi^{*}+\frac{\alpha}{\sigma^{1 / 2}} \theta_{e 1}^{\prime}(0)\left(\frac{7}{8}-u^{*}\right)\right] \frac{d u^{*}}{d \xi^{*}}-u^{*}=0 ;
$$

it is easy to show that, $\alpha \sigma^{-1 / 2}$ has a maximum value of 1.76, for $A_{i}=2.5$, at $\alpha=0.039$.

$$
\text { For } \xi^{*} \rightarrow \pm \infty \text {, we find }
$$

$$
u^{*} \simeq a^{ \pm}\left( \pm \xi^{*}\right)^{1 / 8} \text { 。 }
$$

Matching to the solution of the inner layer for $\hat{\xi}-1 / 2$ $-0^{ \pm}$, yields, from $\mathrm{Eq}$. (31),

$$
a^{*}=-\left[\alpha \sigma \theta_{e 1}(0)\right]^{1 / 16} C / \theta_{e 1}^{\prime}(0)
$$

$$
a^{-}=\left[16 \sigma / \alpha \theta_{e 1}(0)\right]^{1 / 16} .
$$

Equation (A1) together with the boundary conditions (A2), (A3) may now be solved numerically for any given $\alpha$.

\section{APPENDIX B}

To study the cold, highly dense, narrow layer around $\bar{\eta}$, for $\alpha \gg 1$, we define

$$
z=z, \quad J=\nu(4 \eta-3 y), \quad \hat{P}=\nu z \text { 。 }
$$

Then, system (19), to lowest order but retaining electron conduction, becomes

$$
\begin{aligned}
& 4 \hat{P}=z \frac{d J}{d \eta}, \\
& 3 \frac{d \hat{P}}{d \eta}=10 J-\frac{d}{d \eta}\left(\frac{J^{2} z}{\hat{P}}+\eta J\right), \\
& \frac{z^{13 / 3}}{\hat{P}^{4 / 3}} \frac{d}{d \eta}\left(\frac{J \hat{P}^{4 / 3}}{z^{10 / 3}}\right)=\left(\frac{3}{8}\right)^{5 / 2} \frac{\gamma^{3}}{2 \alpha^{3 / 2}} \frac{d}{d \eta} z^{5 / 2} \frac{d z}{d \eta} .
\end{aligned}
$$

In the isentropic solution $(\eta>\bar{\eta})$,

$$
J \simeq \frac{26}{5} B_{2}(\eta-\vec{\eta})^{10 / 13},
$$

which is $O\left(\alpha^{-3 / 7}\right)$ for $\eta-\bar{\eta}=O\left(\alpha^{-39 / 70}\right)$. Defining a point $\eta_{1}$ and a new variable $\hat{\eta}$

$$
\eta_{1}=\bar{\eta}+b \alpha^{-39 / 70}, \quad \hat{\eta}=\alpha^{\gamma}\left(\eta-\eta_{1}\right) \quad(r>39 / 70),
$$

the isentropic solution, near $\eta_{1}$, behaves as

$z \simeq B_{1}(\eta-\bar{\eta})^{3 / 13} \simeq \alpha^{-9 / 70} B_{1} b^{3 / 13}\left(1+\alpha^{-117 / 140} 3 \hat{\eta} / 13\right)+\cdots$, $J \simeq \alpha^{-3 / 7} 5.2 B_{2} b^{10 / 13}\left(1+\alpha^{-117 / 140} 10 \hat{\eta} / 13 b\right)+\cdots$, $\hat{P} \simeq B_{1} B_{2}+\cdots$,

where we have set $r=39 / 28$ to retain heat conduction in (B1) to lowest order.

We next expand the variables in the neighborhood of $\eta_{1}$

$$
\begin{aligned}
& z=\alpha^{-9 / 70} B_{1} b^{3 / 13}+\alpha^{-27 / 28} z_{1}+\cdots, \\
& J=\alpha^{-3 / 7} 5.2 B_{2} b^{10 / 13}+\alpha^{-177 / 140} J_{1}+\cdots, \\
& \hat{P}=B_{1} B_{2}+\cdots,
\end{aligned}
$$

so that (B1) becomes 
$4 B_{2}=b^{3 / 13} d J_{1} / d \hat{\eta}, \quad d \hat{P} / d \hat{\eta}=0$,

$\left(\frac{3}{8}\right)^{5 / 2} \frac{\gamma^{3}}{2} B_{1}^{5 / 2} b^{15 / 26} \frac{d^{2} z_{1}}{d \hat{\eta}^{2}}=4 B_{1} B_{2}-\frac{52}{3} B_{2} b^{10 / 13} \frac{d z_{1}}{d \hat{\eta}} ;$

then

$J_{1}=4 B_{2} \hat{\eta} / b^{3 / 13}+F$,

$\hat{P}=B_{1} B_{2}$,

$z_{1}=G+H \exp \left[-\frac{52}{3}\left(\frac{8}{3}\right)^{5 / 2} \frac{2 b^{5 / 26} B_{2}}{\gamma^{3} B_{1}^{5 / 2}} \hat{\eta}\right]+\frac{3 B_{1} \hat{\eta}}{13 b^{10 / 13}}$

where $F, G, H$ are constants. Clearly, the expansion (B2) ceases to be valid for $-\hat{\eta}=0(\ln \alpha)$.

\section{Defining}

$$
z=\alpha^{-9 / 70} \hat{\hat{z}} \text {, }
$$

Eq. (B1c), to lowest order, becomes

$$
\left(\frac{3}{8}\right)^{5 / 2} \frac{\gamma^{3}}{7} \frac{d^{2} \hat{\hat{E}}^{7 / 2}}{d \hat{\eta}^{2}}+\frac{52}{3} B_{2} b^{10 / 13} \frac{d \hat{\hat{z}}}{d \hat{\eta}}=0
$$

Integrating once, we get

$$
3(3 / 8)^{5 / 2} \gamma^{3} \hat{z}^{5 / 2} d \hat{\hat{z}} / d \hat{\eta}+104 B_{2} b^{10 / 13}\left(\hat{z}-B_{1} b^{3 / 13}\right)=0
$$

whose solution is

$$
\begin{aligned}
-\hat{\eta}= & \left(\frac{3}{8}\right)^{5 / 2} \frac{3}{52} \frac{\gamma^{3}}{B_{2} b^{10 / 13}}\left(\frac{\hat{\hat{z}}^{5 / 2}}{5}+B_{1} b^{3 / 13} \frac{\hat{z}^{3 / 2}}{3}\right. \\
& \left.+B_{1}^{2} b^{6 / 13} \hat{\hat{z}}^{1 / 2}+\frac{B_{1}^{5 / 2} b^{15 / 26}}{2} \ln \frac{\hat{z}^{1 / 2}-B_{1}^{1 / 2} b^{3 / 26}}{\hat{z}^{1 / 2}+B_{1}^{1 / 2} b^{3 / 26}}\right) .
\end{aligned}
$$

Then, for $\hat{\eta} \rightarrow-\infty$

$$
\begin{aligned}
& z=\alpha^{-9 / 70} \hat{\widehat{z}} \simeq \alpha^{-9 / 70}\left(\frac{8}{3}\right)\left(\frac{260}{3} \frac{B_{2} b^{10 / 13}}{\gamma^{3}}\right)^{2 / 5}(-\hat{\eta})^{2 / 5}, \\
& J \simeq \alpha^{-3 / 7} 5.2 B_{2} b^{10 / 13}, \quad \hat{P}=B_{1} B_{2} 。
\end{aligned}
$$

Matching to the solution of the conduction region gives

$$
\begin{aligned}
& b=\left(15 D_{2} B_{1} / 26 D_{1}\right)^{13 / 10} \simeq 0.032, \\
& z_{\min } \simeq \alpha^{-9 / 70} B_{1} b^{3 / 13} \simeq 0.77 \alpha^{-9 / 70}, \\
& \nu_{\max }=\alpha^{9 / 70} B_{2} / b^{3 / 13} \simeq 1.72 \alpha^{9 / 70} .
\end{aligned}
$$

${ }^{1} \mathrm{~J}$. Nuckolls, L. Wood, A. Thiessen, and G. Zimmerman, Nature 239, 139 (1972).

${ }^{2} \mathrm{~J}$. G. Linhart, Nucl. Fusion 10, 211 (1970).

${ }^{3} \mathrm{~K}$. A. Brueckner and S. Jorna, Rev. Mod. Phys. 46, 325 (1974).

${ }^{4} \mathrm{~A}$. Caruso and $\mathrm{R}$. Gratton, Plasma Phys, 10, 867 (1968).

${ }^{5} \mathrm{Yu}$. V. Afanasyev, O.N. Krokhin, and G. V。 Sklizkov, IEEE J. Quantum Electron, QE-2, 483 (1966).

${ }^{6}$ C. Fauquignon and F. Floux, Phys. Fluids 13, 386 (1970).

7J. L. Bobin, Phys. Fluids 14, 2341 (1971).

${ }^{8} \mathrm{~A}$. Caruso and R. Gratton, Plasma Phys, 11, 839 (1969).

${ }^{9} J$. P. Babuel-Peyrissac, C. Fauquignon, and F. Floux, Phys. Lett. A 30, 290 (1969).

${ }^{10} \mathrm{~J}$. Nuckolls, in Laser Interaction and Related Plasma Phenomena (Plenum, New York, 1974), Vol. 3B, p. 399.

${ }^{11}$ J. S. Clarke, H. N. Fisher, and R. J. Mason, Phys. Rev. Lett. 30, 89 (1973).

${ }^{12}$ R. J. Mason and R. L. Morse, Phys. Fluids 18, 814 (1975).

${ }^{13}$ P. Mulser, Z. Naturforsch. A25, 282 (1970).

${ }^{14}$ S. I . Anisimov, Zh. Eksp. Teor. Fiz. Pis'ma Red.12, 414 (1970) [JETP Lett. 12, 287 (1970)],

${ }^{15}$ R. Marshak, Phys. Fluids 1, 24 (1958).

${ }^{16} \mathrm{~L}$. Spitzer, Physics of Fully Ionized Gases (Interscience, New York, 1962).

${ }^{17} \mathrm{~S}$. Braginskii, in Reviews of Plasma Physics (Consultants Bureau, New York, 1965), Vol. 1, p. 205.

${ }^{18}$ R. L. Morse and C. W. Nielson, Phys. Fluids 16, 909 (1973).

${ }^{19}$ Ya. B. Zel'dovich and Yu, P. Raizer, Physics of Shock Waves and High-Temperature Hydrodynamic Phenomena (Academic, New York, 1966), VoI. II, Chaps. VII and X.

${ }^{20}$ R. C. Mjolsness and H. M. Ruppel, Phys. Fluids 15, 1620 (1972).

${ }^{21}$ L. D. Landau and E. M. Lifshitz, Fluid Mechanics (Pergamon, London, 1959), Chap. IX.

${ }^{22}$ L. I. Sedov, Similarity and Dimen sional Methods in Mechanics (Academic, New York, 1959), Chap. IV.

${ }^{23} \mathrm{~K}$. C. Wang, J。 Fluid Mech. 20, 447 (1964)。

${ }^{24}$ J. B. Helliwell, J. Fluid Mech. 37, 497 (1969). 